\title{
Transitive flows on manifolds
}

\author{
Víctor Jiménez López and Gabriel Soler López
}

\begin{abstract}
In this paper we characterize manifolds (topological or smooth, compact or not, with or without boundary) which admit flows having a dense orbit (such manifolds and flows are called transitive) thus fully answering some questions by Smith and Thomas. Namely, it is shown that a surface admits a transitive flow (which can be got smooth) if and only if it is connected and it is neither homeomorphic to the sphere nor the projective plane nor embeddable in the Klein bottle (or, alternatively, if it is connected and includes two orientable topological circles intersecting transversally at exactly one point). We also prove that any (connected) manifold with dimension at least 3 admits a transitive flow, which can be got smooth if the manifold admits a smooth structure.

In particular, this allows us to characterize $\omega$-limit sets with nonempty interior for flows in a given $n$-manifold (as they can be described by the property of being the closure of its transitive $n$-submanifolds).
\end{abstract}

\section{Introduction}

A flow on a manifold $M$ (topological or smooth, compact or not, with or without boundary) is a one-parameter family $\left\{\Phi^{t}\right\}_{t \in \mathbb{R}}$ of homeomorphisms of $M$ such that $\Phi^{t} \circ \Phi^{s}=\Phi^{t+s}$ for any $t, s \in \mathbb{R}$ (we also assume that the $\operatorname{map} \Phi: \mathbb{R} \times M \rightarrow M$ given by $\Phi(t, x)=\Phi^{t}(x)$, which we will identify with the flow in the sequel, is continuous). The orbit of a point $x \in M$ under a flow $\left\{\Phi^{t}\right\}_{t \in \mathbb{R}}$ is the set $\left\{\Phi^{t}(x): t \in \mathbb{R}\right\}$. A flow on a manifold $M$ is said to be (topologically) transitive if there is a point whose orbit under the flow is dense in $M$, and we say that a manifold $M$ is transitive if it admits a transitive flow; if the manifold $M$ admits a (fixed) smooth $\left(C^{\infty}\right)$ structure and $\Phi$ is smooth then we will refer to them as smooth transitive.

2000 Mathematics Subject Classification: Primary 37C70; Secondary 34C05, 37C10. Keywords: manifold, transitive flow. 
The problem of finding transitive flows on manifolds has a long tradition (cf. for instance the bibliography in [16]). Needless to say, the circle is the only transitive 1-manifold. That neither the sphere $\mathbb{S}^{2}$, the projective plane $\mathbb{P}^{2}$ nor the Klein bottle $\mathbb{B}^{2}$ are transitive was more or less known since the seminal work of Bendixson [2] a century ago in the sphere case (and probably in the projective plane case as well), and since at least 1969 [9] in the Klein bottle case. The existence of smooth transitive flows for all other closed surfaces is proved e.g. in [4], although probably it was known rather earlier. In the multidimensional case $(n \geq 3)$ one should refer at least to Oxtoby and Ulam [12], who proved in 1941 that virtually all $n$-dimensional compact connected polyhedra admit (continuous) transitive flows, to a lesser known paper by Sidorov [15] (1968) where it is shown that any region in $\mathbb{R}^{n}$ is smooth transitive (with the standard differential structure) and to Anosov [1] (1974) whose more general result on ergodic flows implies in particular that all compact connected smooth $n$-manifolds (and also $\mathbb{R}^{n}$ ) are smooth transitive.

The task of classifying transitive manifolds was systematically undertaken by Smith and Thomas in 1988 in the papers [16] and [17]. Essentially, they characterize transitive (and, equivalently, smooth transitive) compact connected surfaces as those which cannot be embedded in $\mathbb{S}^{2}, \mathbb{P}^{2}$ or $\mathbb{B}^{2}$, and provide an alternative, simpler proof of the above-mentioned weaker version of Anosov's result: the problems of characterizing which non-compact surfaces and manifolds are transitive are posed as open there.

Up to our knowledge, the last steps in this direction where independently given by Benière in his Ph.D. Thesis [3] (1998) and the second author in his Master Thesis [18] (1999). In [3] it is proved that all connected orientable surfaces without boundary which are not embeddable in $\mathbb{S}^{2}$ are smooth minimal and hence smooth transitive. Alternatively, smooth transitive connected surfaces without boundary were characterized (under the additional assumption of finite genus) in [18] as those including two "crossing circles", that is, two orientable simple closed curves intersecting transversally at exactly one point.

In this paper we culminate the classification of transitive manifolds by proving first:

Theorem A. Let $S$ be a connected surface (resp. a connected orientable surface). Then the following statements are equivalent:

(i) $S$ is smooth transitive;

(ii) $S$ is transitive;

(iii) $S$ is not homeomorphic to $\mathbb{S}^{2}, \mathbb{P}^{2}$, nor to any surface in $\mathbb{B}^{2}$ (resp. is not homeomorphic to any surface in $\left.\mathbb{S}^{2}\right)$;

(iv) S has two crossing circles. 
Remark 1.1. In the orientable case much of Theorem A follows of course from Beniére's result, but some rather strong tools are required (Falconer's results on Hausdorff measures and interval exchange transformations) which are unnecessary for our purposes. Thus we have decided to include a rather elementary and complete proof of Theorem A below.

We also prove:

Theorem B. Let $M$ be a connected $n$-manifold (resp. a connected smooth $n$-manifold), $n \geq 3$. Then it is transitive (resp. smooth transitive).

Recall that if $x \in M$ and $\Phi$ is a flow on a $n$-manifold $M$ then the $\omega$-limit set of the orbit of $x$ under the flow $\Phi$ is defined by

$$
\omega_{\Phi}(x)=\left\{y \in X: \text { there is a sequence }\left(t_{m}\right)_{m} \rightarrow \infty \text { with }\left(\Phi^{t_{m}}(x)\right)_{m} \rightarrow y\right\}
$$

(the $\alpha$-limit set of the orbit of $x, \alpha_{\Phi}(x)$, is analogously defined just replacing $\infty$ by $-\infty)$. If, moreover, $\omega_{\Phi}(x)$ has nonempty interior $O$, then $O$ is connected and invariant for the flow $\Phi$ (in particular it includes the orbit of $x$ ) and has $\omega_{\Phi}(x)$ as its closure: see Lemma 2.2. Hence, $\Phi$ can be seen as a transitive flow on $O$, which is then a transitive $n$-submanifold of $M$. Conversely, let $T$ be a transitive $n$-submanifold of $M$ and let $\Phi$ be the corresponding transitive flow on $T$ (then $T$ is an $\omega$-limit set for $\Phi$ by Lemma 2.4). As all maps $\Phi^{t}$ are homeomorphisms and, because of the theorem of invariance of domain [8, p. 475], any subset of $M$ which is homeomorphic to an open set of $\mathbb{R}^{n}$ is open as well, the combinatorial boundary $\partial T$ of $T$ is invariant for $\Phi$. Then we can see $\Phi$ as a flow on the open set $T \backslash \partial T$ and extend it via Lemma 2.3 to a flow on $M$ still having $T$ as one of its $\omega$-limit sets. Thus, $\omega$-limit sets (and, similarly, $\alpha$-limit sets) with nonempty interior in $M$ are exactly the closure of its transitive $n$-submanifolds and Theorems $\mathrm{A}$ and $\mathrm{B}$ allow us to characterize them.

\section{Preliminaries}

Let $X$ be a metrizable space (much of the ensuing discussion also works in less restrictive settings, but this one is general enough for our purposes). We call a continuous map $\Phi: \Lambda \subset \mathbb{R} \times X \rightarrow X$ a local flow on $X$ provided that the following properties hold:

(i) $\Lambda$ is open in $\mathbb{R} \times X$; moreover, for any $x \in X$ the set of numbers $t$ for which $\Phi(t, x)$ is defined is an open interval $I_{x} \ni 0$;

(ii) $\Phi(0, x)=x$ for any $x \in X$;

(iii) if $\Phi(t, x)=y$ then $I_{y}=\left\{s-t: s \in I_{x}\right\}$; moreover, $\Phi(u, y)=$ $\Phi(u, \Phi(t, x))=\Phi(u+t, x)$ for any $u \in I_{y}$. 
If we are in the particular case $\Lambda=\mathbb{R} \times X$ then we get a flow on $X$, as we did in the Introduction in the case of manifolds. Notice that if $X$ is compact then $I_{x}=\mathbb{R}$ for any $x \in X$ so any local flow is in fact a flow. We use the notation $\Phi(t, x)=\Phi^{t}(x)=\Phi_{x}(t)$ whenever it makes sense, being $\Phi_{x}\left(I_{x}\right)$ the orbit of a point $x$ under the local flow $\Phi$. We say that a point $x \in X$ is singular if its orbit consists of just the point $x$.

Of course we are primarily interested in manifolds, and local flows naturally arise there as associated to autonomous systems of differential equations (thus the need of introducing this otherwise artificial notion). More precisely, it is well known that if $\Phi: \Lambda \rightarrow M$ is a smooth local flow then there is a smooth vector field $F: M \rightarrow T M$ (its associated vector field) such that $\frac{\partial \Phi}{\partial t}(t, x)=F(\Phi(t, x))$ for any $t$ and $x$ and that, conversely, for any smooth vector field $F$ on $M$ there is a smooth local flow $\Phi$ on $M$ (its associated local flow) such that $\frac{\partial \Phi}{\partial t}(t, x)=F(\Phi(t, x))$ for any $t$ and $x$.

When we speak about an n-manifold (or sometimes just a manifold) we refer to a Hausdorff topological space with a countable base (usually denoted by $M$ ) which is locally homeomorphic to the closed ball $\left\{x \in \mathbb{R}^{n}\right.$ : $\|x\| \leq 1\}$ (hence it need not be compact and may have (combinatorial) boundary points, which are those having no neighbourhood homeomorphic to the open ball $\left.\left\{x \in \mathbb{R}^{n}:\|x\|<1\right\}\right)$. As usual, if $M$ is an $n$-manifold then we denote by $\partial M$ the set of its boundary points. If $n=2$ then $M$ is called a surface and we will often use the symbol $S$ to denote it; in the particular case when $S$ is compact, connected and has empty boundary we call it a closed surface. As it is well known, all $n$-manifolds with $n \leq 3$ admit a smooth structure which is unique up to diffeomorphisms (cf. Theorem 2.1), but if $n>3$ they may admit one, none or even infinitely many. Thus, when speaking about a smooth manifold in this setting, we will implicitly assume that we have fixed a smooth structure on it.

If $X$ is metrizable and $A \subset X$ then $\mathrm{Cl} A, \operatorname{Int} A, \operatorname{Bd} A$ and $\operatorname{diam} A$ will denote the closure, the interior, the boundary of $A$ and the diameter (for a fixed distance $d(\cdot, \cdot)$ in $X$ ) of $A$, and if $f$ is a map defined on $X$, then $\left.f\right|_{A}$ will denote its restriction to $A$. Notice that "boundary" refers now to topological boundary, as opposed to "combinatorial boundary" before (in the rest of the paper it should be clear what kind of "boundary" we are referring to

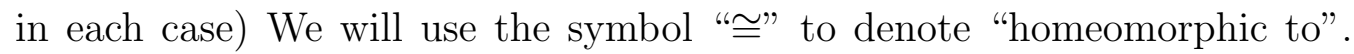
A continuous map $f: X \longrightarrow Y$ is called an embedding if $f$ maps $X$ homeomorphically onto $f(X)$; in this situation we also say that $X$ is embeddable or can be embedded in $Y$. If additionally $X$ and $Y$ are manifolds and $f$ maps diffeomorphically $X$ onto $f(X)$ then we say that $f$ is a smooth embedding. 
We will denote by $\|\cdot\|$ the euclidean norm in $\mathbb{R}^{n}$, when $\mathbb{O}^{n}(\rho)=\{x \in$ $\left.\mathbb{R}^{n}:\|x\|<\rho\right\}, \mathbb{D}^{n}(\rho)=\left\{x \in \mathbb{R}^{n}:\|x\| \leq \rho\right\}$, will be the corresponding open and closed balls of radius $\rho$. We will rewrite $\mathbb{O}^{n}:=\mathbb{O}^{n}(1), \mathbb{D}^{n}:=\mathbb{D}^{n}(1)$ and put $\mathbb{S}^{n}=\left\{x \in \mathbb{R}^{n+1}:\|x\|=1\right\}$ as usual. Also, let $\mathbb{T}^{2}=\mathbb{S}^{1} \times \mathbb{S}^{1}$, $\mathbb{P}^{2}=\mathbb{S}^{2} / \sim, \mathbb{M}^{2}=\left(\mathbb{S}^{1} \times(-1,1)\right) / \sim($ with $x \sim y$ if $x= \pm y$ in both cases) and $\mathbb{B}^{2}=\left(\mathbb{S}^{1} \times[-1,1]\right) / \sim$ (here " " identifies the points $(t, 1)$ and $(-t,-1)$ for any $\left.t \in \mathbb{S}^{1}\right)$. We call an open arc (resp. an arc, a circle, a disk, a closed disk, a sphere, a torus, a projective plane, a Klein bottle, a Möbius band, an annulus, a closed annulus, an $n$-cell and an $n$-sphere) any space homeomorphic to an open interval (resp. a compact interval, $\mathbb{S}^{1}, \mathbb{O}^{2}, \mathbb{D}^{2}$, $\mathbb{S}^{2}, \mathbb{T}^{2}, \mathbb{P}^{2}, \mathbb{B}^{2}, \mathbb{M}^{2}, \mathbb{S}^{1} \times(-1,1), \mathbb{S}^{1} \times[-1,1], \mathbb{D}^{n}$ and $\left.\mathbb{S}^{n}\right)$. Notice that we are using "arc" and "circle" instead of the more usual, but longer terms, "simple curve" and "simple closed curve".

When denoting a circle by a greek letter, say $\alpha$, we will use the symbol to simultaneously refer to the circle itself and a (fixed) homeomorphism mapping $\mathbb{S}^{1}$ onto the circle. For instance, if $\alpha$ and $\beta$ are the components of the boundary of a closed annulus $A$ and we say that they have compatible orientations then we mean that there is a homeomorphism $h: \mathbb{S}^{1} \times[-1,1] \rightarrow$ $A$ such that $h(t,-1)=\alpha(t)$ and $h(t, 1)=\beta(t)$ (this amounts to say that both circles are either clockwise oriented or counterclockwise oriented by the homeomorphisms $\alpha$ and $\beta$ after seeing $A$ as a plane subset). Similarly, we say that two disjoint circles $\alpha, \beta \subset \mathbb{S}^{2}$ have compatible orientations if they have compatible orientations with regard to the closed annulus $\mathbb{S}^{2} \backslash\left(D_{\alpha} \cup D_{\beta}\right)$, where $D_{\alpha}$ and $D_{\beta}$ are the disjoint disks enclosed by $\alpha$ and $\beta$. If $\alpha \subset X$ is a circle then we will say that $\alpha$ is orientable (resp. nonorientable) if it admits a neighbourhood homeomorphic to the annulus $\mathbb{S}^{1} \times(-1,1)$ (resp. the Möbius band $\mathbb{M}^{2}$ ) with the central circle being mapped by the homeomorphism onto $\alpha$. It is well known that any circle in a surface $S$ is either orientable or nonorientable (after we include among orientable circles those which are components of $\partial S$, as they admit a neighbourhood homeomorphic to a closed annulus). A surface admitting only orientable circles is called orientable; otherwise it is nonorientable.

And finally, we say that two circles $\alpha, \beta \in X$ intersect transversally at $x$ if $x \in \alpha \cap \beta$ and there is a neighbourhood (a disk) $D$ of $x$ such that both $\gamma=\alpha \cap D$ and $\delta=\beta \cap D$ are open arcs, they decompose $D$ into two disks $D_{1}^{\gamma}, D_{2}^{\gamma}$ and $D_{1}^{\delta}, D_{2}^{\delta}$, and they are decomposed by $x$ into two open $\operatorname{arcs} \gamma_{1}, \gamma_{2}$ and $\delta_{1}, \delta_{2}$ with $\delta_{i} \subset D_{i}^{\gamma}$ and $\gamma_{i} \subset D_{i}^{\delta}, i=1,2$. If two orientable circles intersect (transversally) at exactly one point then we refer to them as two crossing circles. 
We conclude this section stating and proving some previously cited results.

Theorem 2.1. Any $n$-manifold $(n \leq 3)$ admits a (unique up to diffeomorphisms) smooth structure. In particular, if $M$ and $N$ are homeomorphic $n$-manifolds $(n \leq 3)$ then they are diffeomorphic.

Proof. See for instance Example 3.1.6 from [19, p. 112] (the restrictions $\partial M=\partial N=\emptyset$ there are not essential).

Lemma 2.2. Let $\Phi$ be a flow on a metrizable space $X$ and let $x \in X$. Assume that $O=\operatorname{Int} \omega_{\Phi}(x) \neq \emptyset$. Then $O$ is invariant for $\Phi$ (in particular $\left.\Phi_{x}(\mathbb{R}) \subset O\right), O$ is connected and $\mathrm{Cl} O=\omega_{\Phi}(x)$.

Proof. The orbit $\Phi_{x}(\mathbb{R})$ must intersect $O$ at some point $y$. Since $\omega_{\Phi}(x)$ is invariant under $\Phi$ then $\Phi(\mathbb{R} \times O) \subset \omega_{\Phi}(x)$; in particular, $\Phi_{x}(\mathbb{R})=\Phi_{y}(\mathbb{R}) \subset$ $\omega_{\Phi}(x)$. Notice that $\Phi(\mathbb{R} \times O)$ is open (because it is the union of all sets $\Phi^{t}(O)$, which are open since $\Phi^{t}$ is a homeomorphism for every $t$ ), connected and dense in $\omega_{\Phi}(x)$ (these last two properties because it includes the orbit $\left.\Phi_{x}(\mathbb{R})\right)$. Observe that $\Phi(\mathbb{R} \times O)=O$ and then we are done.

Lemma 2.3. Let $Y$ be a locally compact metrizable space and let $X \subset Y$ be open. Let $\Phi: \Lambda \rightarrow X$ be a local flow on $X$. Then there is a flow $\Psi$ on $Y$ for which all points from $Y \backslash X$ are singular and having the same orbits as those of $\Phi$ in $X$. Moreover, for any $x \in X$ the bijection $\theta_{x}: I_{x} \rightarrow \mathbb{R}$ satisfying $\Phi_{x}(t)=\Psi_{x}\left(\theta_{x}(t)\right)$ for any $t$ is increasing.

Moreover, if $Y$ is a smooth manifold and $\Phi$ is smooth on $X$, then the flow $\Psi$ can be got smooth in $Y$.

Proof. We will first prove the lemma in its continuous version. To begin with, notice that it is not restrictive to assume that $Y$ is connected (as if $Z$ is an arbitrary component of $Y$ then the open subset $X \cap Z$ of $Z$ is a union of orbits of $\Phi$, that is, the restriction of $\Phi$ to $\Lambda \cap(\mathbb{R} \times(X \cap Z))$ is a local flow on $X \cap Z)$. Moreover, since $Y$ is locally compact and metrizable we have then that $Y$ has a countable base (see [11, pp. 257 (Theorem 41.4) and 261 (Exercise 10)]); hence, $Y_{\infty}$ is metrizable as well [8, p. 43, Theorem 5].

Fix a distance $d$ in $Y_{\infty}$ for which there is some $y \in X$ such that $d(y, \operatorname{Bd} X)>1$ (here $\operatorname{Bd} X$ is the boundary of $X$ in $Y_{\infty}$ ) and find positive numbers $t_{n}, n=1,2, \ldots$, small enough so that if $d(x, \operatorname{Bd} X) \geq 1 / n$ then $d(\Phi(t, x), \operatorname{Bd} X) \geq 1 /(n+1)$ for any $|t| \leq t_{n}$. Fix a continuous map $\Theta: X \rightarrow$ $(0, \infty)$ satisfying $\Theta(x) \geq 1 / t_{n}$ for any $x$ with $d(x, \operatorname{Bd} X) \geq 1 /(n+1)$. Now, for any $\theta \in \mathbb{R}$ and $x \in X$ there is exactly one number $t(\theta, x):=t \in I_{x}$ such that $\theta=\int_{0}^{t} \Theta(\Phi(s, x)) d s$. It is simple to check that $t(\theta, x)$ is continuous and, then, that $\Psi(\theta, x)=\Phi(t(\theta, x), x)$ is a flow on $X$ which can be continuously extended to the rest of $Y_{\infty}$ (in particular to $Y$ ) by writing $\Psi(\theta, x)=x$ for any $\theta \in \mathbb{R}$ and any $x \in Y_{\infty} \backslash X$. 
Now we generalize the ideas from [16, Lemma 2.1] to prove the smooth version of the lemma. See $Y$ as an $n$-submanifold of $\mathbb{R}^{m}$ for $m=2 n$. We can suppose that $Y$ is bounded in $\mathbb{R}^{m}$ and hence that $K=(\mathrm{Cl} Y) \backslash X$ is compact. Fix a collection $\left\{U_{i}\right\}_{i=1}^{\infty}$ of open bounded neighbourhoods of $K$ in $\mathbb{R}^{m}$ such that $\mathrm{Cl} U_{i+1} \subset U_{i}$ for any $i$ and $\bigcap_{i=1}^{\infty} U_{i}=K$. Find smooth maps $\lambda_{i}: \mathbb{R}^{m} \rightarrow[0,1]$ satisfying $\lambda_{i} \equiv 1$ on $\mathbb{R}^{m} \backslash U_{i}$ and $\lambda_{i} \equiv 0$ on $\mathrm{Cl} U_{i+1}$ and consider the (clearly well defined and smooth) vector fields $F_{i}: Y \rightarrow \mathbb{R}^{m}$ given by

$$
F_{i}(x)= \begin{cases}\lambda_{i}(x) F(x) & \text { if } x \in X, \\ 0 & \text { otherwise }\end{cases}
$$

where $F: X \rightarrow \mathbb{R}^{m}$ is the smooth vector field associated to $\Phi$.

Take a countable atlas $\left\{\varphi_{i}\right\}_{i=1}^{\infty}, \varphi_{i}: V_{i} \subset \mathbb{R}^{n} \rightarrow Y$. It is not restrictive to assume that, if $\left\{\epsilon_{i}\right\}_{i=1}^{\infty}$ are appropriately chosen small positive numbers and we write $G_{i}(x)=\epsilon_{i} F_{i}(x)$, then, for any $j$, we have that the compositions $G_{j} \circ \varphi_{i}, i=1,2, \ldots, j$, have all its partial derivatives up to the order $j$ uniformly bounded by $1 / 2^{j}$. Then it is clear that $G=\sum_{i=1}^{\infty} G_{i}$ is a smooth vector field in $Y$ vanishing at $Y \backslash X$ and whose associated local flow $\Psi$ has the same orbits, with the same orientations, as those of $\Phi$. Moreover, we can assume that the choosing of the numbers $\epsilon_{i}$ guarantees that $\|G(x)\|<d\left(\mathbb{R}^{m} \backslash\right.$ $\left.U_{i}, \mathrm{Cl} U_{i+1}\right)$ (with $d(\cdot, \cdot)$ the euclidean distance) for any $x \in X \cap\left(U_{i} \backslash U_{i+1}\right)$. Now, it is obvious that $\Psi$ is in fact a flow and we have finished.

Lemma 2.4. Let $\Phi$ be a flow on a locally compact metrizable space $X$. Then the following statements are equivalent:

(i) there is a point $x \in X$ whose orbit is dense in $X$;

(ii) there is a $G_{\delta}$ dense set of points each of them having $X$ as both its $\omega$-limit and its $\alpha$-limit set.

Proof. We only have to prove (i) $\Rightarrow($ ii). As $X$ is a Baire space [11, p. 299], we just have to find open dense sets $\left\{O_{n}\right\}_{n=1}^{\infty}$ in $X$ such that $\alpha_{\Phi}(y)=\omega_{\Phi}(y)=X$ for any $y \in \bigcap_{n=1}^{\infty} O_{n}$.

Notice that $X$ is connected and thus has a countable base $\left\{U_{n}\right\}_{n=1}^{\infty}$ of open sets. Take $x \in X$ with $\mathrm{Cl} \Phi_{x}(\mathbb{R})=X$. Fix any pair $U_{m}$ and $U_{n}$ of these sets. Then there are points $a=a_{m, n}$ and $z=z_{m, n}$ in $\Phi_{x}(\mathbb{R}) \cap U_{m}$ and numbers $t_{a}<-n$ and $t_{z}>n$ with $\Phi_{a}\left(t_{a}\right), \Phi_{z}\left(t_{z}\right) \in U_{n}$ (and then $\Phi_{u}\left(t_{a}\right), \Phi_{v}\left(t_{z}\right) \in U_{n}$ for any $u \in W_{a}$ and $v \in W_{z}$ for some small neighbourhoods $W_{a}$ and $W_{z}$ of $a$ and $z$ ). Now it suffices to take

$$
O_{n}=\left(\bigcup_{m=1}^{\infty} W_{a_{m, n}}\right) \bigcap\left(\bigcup_{m=1}^{\infty} W_{z_{m, n}}\right) .
$$




\section{Proof of Theorem A}

A few words about the proof. After some preliminary work we will show that we can restrict ourselves to the empty boundary case (Lemma 3.4). Then, to prove (iv) $\Rightarrow$ (i) (the implications (i) $\Rightarrow$ (ii), (ii) $\Rightarrow$ (iii) and (iii) $\Rightarrow$ (iv) are easy or even trivial) we will proceed as follows. First we show that there is a dense region $O \subset S$, still having two crossing circles, which can be embedded in $\mathbb{T}^{2}$.

Next we prove that $O$ is homeomorphic to $\mathbb{T}^{2} \backslash A$, with $A$ being a totally disconnected set which is included in an arc of constant irrational slope.

Finally we slightly alter the corresponding irrational flow in $\mathbb{T}^{2}$ so that $A$ is exactly the set of critical points of the new flow (which, in particular, has a dense orbit in $\mathbb{T}^{2} \backslash A$ ), apply Theorem 2.1 to carry this flow to a smooth transitive flow on $O$, and use Lemma 2.3 to get the desired transitive flow on $S$.

Before going into the details we need to recall some topological results on surfaces that will be used later. Throughout the section we will use liberally, without further reference, some intuitively obvious (but deep) results on the sphere. Among them: a circle decomposes the sphere into two disks, each of them having the circle as its boundary (the Jordan curve theorem); any homeomorphism between two circles in a sphere can be extended to a homeomorphism on the whole sphere (the Schönflies theorem); if we take off the interiors of two disjoint closed disks from the sphere then we get a closed annulus (the two-dimensional annulus theorem); no arc separates the sphere; and so on (a good general reference is [8, Chapter 10]). In fact, the previously stated property for any circle in a surface of being either orientable or nonorientable follows elementary (if rather tediously) from these results (plus of course the fact that a surface is locally euclidean).

Recall that two closed surfaces are homeomorphic if and only if they have the same genus and Euler characteristic. In what follows $M_{g}$ and $N_{g}$ will denote (appropriately fixed) orientable and nonrientable surfaces of genus $g$. Thus $M_{0} \cong \mathbb{S}^{2}, M_{1} \cong \mathbb{T}^{2}, N_{1} \cong \mathbb{P}^{2}$ and $N_{2} \cong \mathbb{B}^{2}$ but the corresponding equalities need not happen. Recall that $\chi\left(M_{g}\right)=2-2 g$ and $\chi\left(N_{g}\right)=2-g$, where $\chi(S)$ denotes the Euler characteristic of $S$ (that is, $\chi(S)=F+V-E$ with $F, V$ and $E$ being the number of faces, vertexes and edges of an arbitrary triangulation of $S$ ).

We next describe a typical procedure to generate all posible closed (and, indeed, non-closed) surfaces. Let $\left\{\alpha_{i}, \beta_{i}\right\}_{i=1}^{i_{0}},\left\{\gamma_{j}\right\}_{j=1}^{j_{0}}, 0 \leq i_{0}, j_{0} \leq \infty$, be countable families (notice that they may be empty and/or infinite) of circles in $\mathbb{S}^{2}$. Assume additionally that the following properties hold $(\Sigma=$ $\left.\left\{\alpha_{i}, \beta_{i}\right\}_{i=1}^{i_{0}} \cup\left\{\gamma_{j}\right\}_{j=1}^{j_{0}}\right)$ : 
(i) the circles $\sigma \in \Sigma$ are pairwise disjoint and enclose pairwise disjoint disks $D_{\sigma}$;

(ii) the circles $\alpha_{i}, \beta_{i}$ have compatible orientations for any $i$;

(iii) $d\left(D_{\sigma}, \bigcup_{\sigma^{\prime} \neq \sigma} D_{\sigma^{\prime}}\right)>0$;

(iv) if $i_{0}=\infty$ then $\lim _{i \rightarrow \infty} d\left(D_{\alpha_{i}}, D_{\beta_{i}}\right)=0$;

(v) if $i_{0}=\infty\left(\right.$ resp. $\left.j_{0}=\infty\right)$ then $\lim _{i \rightarrow \infty} \operatorname{diam} D_{\alpha_{i}}=\lim _{i \rightarrow \infty} \operatorname{diam} D_{\beta_{i}}=$ 0 (resp. $\lim _{j \rightarrow \infty} \operatorname{diam} D_{\gamma_{j}}=0$ ).

Define in $\mathbb{S}^{2} \backslash \bigcup_{\sigma \in \Sigma} D_{\sigma}$ the following equivalence relation: $x \sim y$ if and only if either:

- $x=y$;

- $x=\alpha_{i}(t), y=\beta_{i}(t)$ (or $\left.y=\alpha_{i}(t), x=\beta_{i}(t)\right)$ for some $t \in \mathbb{S}^{1}$ and some $i$;

- $x=\gamma_{j}(t), y=\gamma_{j}(-t)$ for some $t \in \mathbb{S}^{1}$ and some $j$.

We call the compact, connected (indeed even metrizable by the NagataSmirnov theorem) quotient space $\mathbb{S}_{\Sigma}^{2}:=\mathbb{S}^{2} / \sim$ a pseudosurface. Notice if $[A]=[A]_{\Sigma}$ denotes the set of equivalence classes under $\sim$ including points from a set $A \subset \mathbb{S}^{2}$, then $[\sigma]$ is a circle in $\mathbb{S}_{\Sigma}^{2}$ for any $\sigma \in \Sigma$, which is orientable in the case $\sigma=\alpha_{i}$ or $\sigma=\beta_{i}$, and nonorientable in the case $\sigma=\gamma_{j}$. It turns out that if $i(\Sigma):=i_{0}$ and $j(\Sigma):=j_{0}$ are both finite then $\mathbb{S}_{\Sigma}^{2}$ is a closed surface, with $\mathbb{S}_{\Sigma}^{2} \cong M_{i(\Sigma)}$ in the case $j(\Sigma)=0$ and $\mathbb{S}_{\Sigma}^{2} \cong N_{2 i(\Sigma)+j(\Sigma)}$ if $j(\Sigma)>0$.

A nice thing about pseudosurfaces is that non-closed surfaces can also be embedded into them [14]:

Theorem 3.1. Let $S$ be a connected surface, $\partial S=0$. Then there is a embedding e $: S \rightarrow \mathbb{S}_{\Sigma}^{2}$ for some collection of circles $\Sigma$ such that $\mathbb{S}_{\Sigma}^{2} \backslash e(S)$ is totally disconnected (that is, each of its components consists of one point) and intersects none of the circles $[\sigma], \sigma \in \Sigma$. Moreover, for any $\epsilon>0$ the maximum distance from the points of the circles $[\sigma]$ to $\mathbb{S}_{\Sigma}^{2} \backslash e(S)$ is less than $\epsilon$ (except at most for finite number of circles).

The next result is essentially proved e.g. in [6, Lemma 2.4]. We will only need to use it in some particular cases, but for completeness we state it in its full generality. Recall that a circle $C \subset X$ is null homotopic if there is a continuous map $H:[0,1] \times[0,1] \rightarrow X$ satisfying $H([0,1] \times\{0\})=C$ and $H(0, s)=H(1, s)=H(t, 1)=x$ for some $x \in C$ and every $t, s \in[0,1]$. 
Lemma 3.2. Let $S$ be a closed surface, let $\alpha \subset S$ be a circle and let $g$ be the genus of $S$.

(i) If $\alpha$ is nonorientable (thus $S \cong N_{g}$ ) then either $S \backslash \alpha \cong M_{(g-1) / 2} \backslash D$ or $S \backslash \alpha \cong N_{g-1} \backslash D$ for any closed disk $D$ in the corresponding surface.

(ii) If $\alpha$ is orientable and $S \backslash \alpha$ is connected then $S \backslash \alpha \cong M_{g-1} \backslash\left(D_{1} \cup D_{2}\right)$ (if $\left.S \cong M_{g}\right)$, and $S \backslash \alpha \cong M_{(g-2) / 2} \backslash\left(D_{1} \cup D_{2}\right)$ or $S \backslash \alpha \cong N_{g-2} \backslash\left(D_{1} \cup D_{2}\right)$ (if $S \cong N_{g}$ ) for any pairwise disjoint closed disks $D_{1}$ and $D_{2}$ in the corresponding surface.

(iii) If $\alpha$ is orientable and non-null homotopic, and $S \backslash \alpha=O_{1} \cup O_{2}$ for some pairwise disjoint open sets $O_{1}$ and $O_{2}$, then there are positive integers $g_{1}, g_{2}$ such that $g_{1}+g_{2}=g$ with $O_{i} \cong M_{g_{i}} \backslash D_{i}, i=1,2$ (if $S \cong M_{g}$ ), and such that $2 g_{1}+g_{2}=g$ with $O_{1} \cong M_{g_{1}} \backslash D_{1}, O_{2} \cong N_{g_{2}} \backslash D_{2}$, or $g_{1}+g_{2}=g$ with $O_{i} \cong N_{g_{i}} \backslash D_{i}, i=1,2$ (if $S \cong N_{g}$ ), for any closed disks $D_{i}, i=1,2$, in the corresponding surfaces.

Moreover, if in cases (ii) and (iii) $\alpha \subset U \subset S$ is a closed annulus which is decomposed by $\alpha$ into two closed annuli $U_{i}, i=1,2$, then the restriction of the corresponding homeomorphism $f$ with domain $S \backslash \alpha$ to $U_{i} \backslash \alpha$ can be extended (after renaming if necessary the closed disks $D_{i}$ ) to a homeomorphism $f_{i}$ : $U_{i} \rightarrow f\left(U_{i} \backslash \alpha\right) \cup \operatorname{Bd} D_{i}, i=1,2$.

Remark 3.3. To complete Lemma 3.2 let us emphasize that if $\alpha$ is a null homotopic circle in $S$ then there is a closed disk $D \in S$ with $\operatorname{Bd} D=\alpha$ (in particular, any null homotopic circle is orientable).

After this preparatory work we are ready to prove Theorem A. Let us first emphasize that:

Lemma 3.4. Theorem A holds for every surface if and only if it holds for all surfaces with empty boundary.

Proof. It suffices to prove that, under the assumption that Theorem A holds true in the empty boundary case, a surface $S$ satisfies (i) (resp. (ii), (iii) or (iv)) in the statement of Theorem $\mathrm{A}$ if and only if $R=S \backslash \partial S$ satisfies (i) (resp. (ii), (iii) or (iv)). Indeed, for properties (i) and (ii) our claim follows from Lemma 2.3, and for property (iv) from the fact that if $S$ has two crossing circles then they can be easily modified to get two crossing circles in $R$ (using for instance that, according to the collaring theorem [5, pp. 113-114], there is an embedding $e: \partial S \times[0,1) \rightarrow S$ with $e(x, 0)=x$ for any $x \in \partial S$ ).

We must be a bit more careful regarding property (iii). Clearly, it suffices to show that if $R$ is embeddable in $\mathbb{B}^{2}$ (resp. in $\mathbb{S}^{2}$ ) then $S$ is embeddable in $\mathbb{B}^{2}$ (resp. in $\mathbb{S}^{2}$ ) as well. 
To do this we use again the collaring theorem to embed $S$ in a surface with empty boundary $T$ (which is orientable if $S$ is orientable) so that $T \backslash S \cong$ $\partial S \times(0,1)$. Now it is simple to check that if $\alpha, \beta$ are two crossing circles in $T$ then they both must intersect $R$ and hence can be modified to get two crossing circles in $R$. Since $R$ cannot have two crossing circles (recall that we are assuming that Theorem A holds true in the empty boundary case), $T$ cannot have them either and we can apply Theorem A to the surface without boundary $T$ to conclude that it is embeddable in $\mathbb{B}^{2}$ (resp. in $\mathbb{S}^{2}$ ). Hence $S$ is embeddable in $\mathbb{B}^{2}$ (resp. in $\mathbb{S}^{2}$ ) as we desired to show.

Thus we will prove Theorem A under the additional assumption $\partial S=\emptyset$. The statement (i) $\Rightarrow$ (ii) is trivial, and (ii) $\Rightarrow$ (iii) follows from the fact that all $\omega$-limit sets for flows in $\mathbb{S}^{2}, \mathbb{P}^{2}$ or $\mathbb{B}^{2}$ have empty interior (see e.g. [17]).

We next prove (iii) $\Rightarrow$ (iv) by showing that if $S$ has no crossing circles then it is homeomorphic to either $\mathbb{S}^{2}, \mathbb{P}^{2}$ or a region in $\mathbb{B}^{2}$ (or, if $S$ is orientable, to a region in $\mathbb{S}^{2}$ ).

Consider the embedding $e: S \rightarrow \mathbb{S}_{\Sigma}^{2}$ from Theorem 3.1. We claim that $i(\Sigma)=0$ and $j(\Sigma) \leq 2$.

Assume $i(\Sigma) \neq 0$. Then for some totally disconnected closed set $C \subset$ $\mathbb{S}^{2} \backslash\left(\bigcup_{\sigma \in \Sigma} \mathrm{Cl} D_{\sigma}\right)$ we have that $S \cong \mathbb{S}_{\Sigma}^{2} \backslash[C]_{\Sigma}$. Moreover, since $C$ is totally disconnected and then cannot separate $\mathbb{S}^{2}$ (use e.g. [8, p. 189 and p. 539, Th. 5]), there is an $\operatorname{arc} \delta \subset \mathbb{S}^{2}$ having each of its endpoints in $\alpha_{1}$ and $\beta_{1}$, respectively, and intersecting no other points from $C \cup \bigcup_{\sigma \in \Sigma} \mathrm{Cl} D_{\sigma}$. Since $\alpha_{1}$ and $\beta_{1}$ have compatible orientations, $[\delta]_{\Sigma}$ and $\left[\alpha_{1}\right]_{\Sigma}=\left[\beta_{1}\right]_{\Sigma}$ are crossing circles in $\mathbb{S}_{\Sigma}^{2} \backslash[C]_{\Sigma}$. Thus $S$ has two crossing circles, a contradiction.

Now we show $j(\Sigma) \leq 2$ (notice that if additionally $S$ is orientable then we automatically get $j(\Sigma)=0$ and $S$ is embeddable in $\mathbb{S}^{2}$ as required). Again we argue by contradiction to find in $S$ three pairwise disjoint nonorientable circles, say $\delta, \phi_{1}$ and $\phi_{2}$ (see Figure 1). Starting from an orientable circle $\zeta$ enclosing $\delta$ it is easy to find two nonorientable circles $\delta_{1}$ and $\delta_{2}$ close to $\delta$ (hence disjoint from $\phi_{1}$ and $\phi_{2}$ ) intersecting transversally at exactly one point $x \in \delta$. Further, there are pairwise disjoint closed disks $K_{1}$ and $K_{2}$ in $S$ such that $K_{l} \cap \delta_{l}=\mu_{l}$ and $K_{l} \cap \phi_{l}=\rho_{l}$ are $\operatorname{arcs}$ in $\mathrm{Bd} K_{l}, l=1,2$. Say $\operatorname{Bd} K_{l}=\mu_{l} \cup \tau_{l, 1} \cup \rho_{l} \cup \tau_{l, 2}$ for appropriate (minimal) arcs $\tau_{l, 1}, \tau_{l, 2}$. Then $\left(\delta_{l} \backslash \mu_{l}\right) \cup \tau_{l, 1} \cup\left(\phi_{l} \backslash \rho_{l}\right) \cup \tau_{l, 2}, l=1,2$, are orientable circles intersecting transversally at $x$, a contradiction.

Thus, $i(\Sigma)=0$ and $j(\Sigma) \leq 2$. This means that $S$ is embeddable in $\mathbb{S}^{2}$, $\mathbb{P}^{2}$ or $\mathbb{B}^{2}$. Moreover, if it is not homeomorphic to any of the first two surfaces then, as any proper region of $\mathbb{S}^{2}$ or $\mathbb{P}^{2}$ is embeddable in $\mathbb{B}^{2}$ (cf. Lemma 3.2(i)), it would be embeddable in $\mathbb{B}^{2}$ as well. The statement (iii) $\Rightarrow$ (iv) is proved.

It only rests to show (iv) $\Rightarrow(\mathrm{i})$. As indicated at the beginning of the section, the proof consists of three steps. 


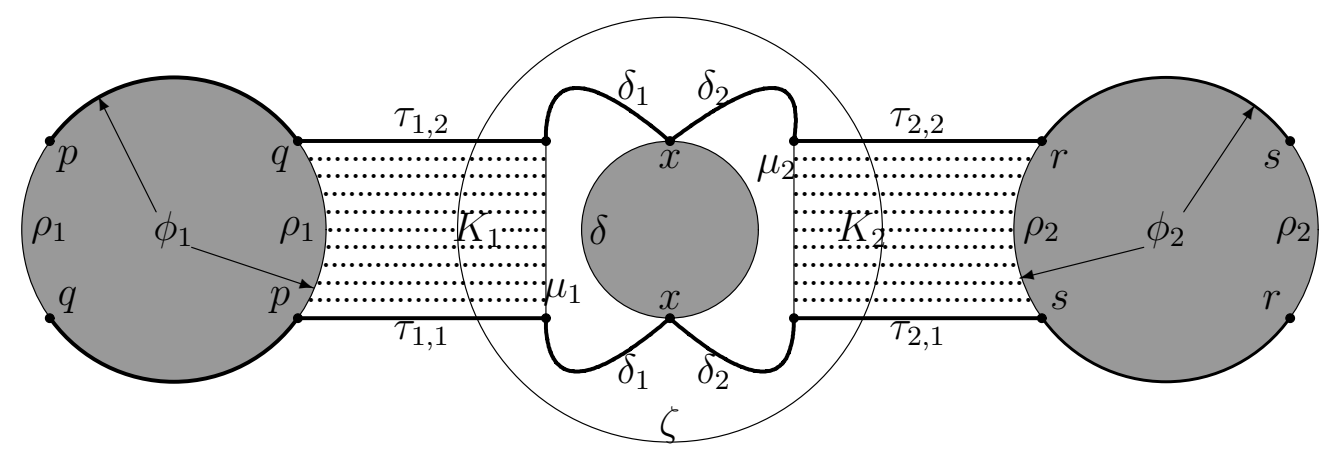

Figure 1: Proof of $j(\Sigma) \leq 2$. Here $\delta, \phi_{1}$ and $\phi_{2}$ are nonorientable Jordan curves, when each point is identified with its opposite as marked for $x, p, q, r$ and $s$.

STEP 1: Finding the region $O$.

As in the proof of (iii) $\Rightarrow$ (iv) we retort to the embedding $e: S \rightarrow \mathbb{S}_{\Sigma}^{2}$ when, according to the reasoning there, we must have either $i(\Sigma) \neq 0$ or $j(\Sigma) \geq 3$. If we are in the case $i(\Sigma) \neq 0$ then it suffices to take off from $S$ all the circles which are mapped by $e$ onto the circles $\left[\alpha_{i}\right]=\left[\beta_{i}\right](i \neq 1)$ and $\left[\gamma_{j}\right]$ to get the desired set $O$ (notice that with the notation we used there, $e^{-1}([\delta]$ ) and $e^{-1}\left(\left[\alpha_{1}\right]\right)$, are crossing circles in $O$; moreover, $O$ is trivially embeddable in $\mathbb{S}_{\Sigma^{\prime}}^{2}, \Sigma^{\prime}=\left\{\alpha_{1}, \beta_{1}\right\}$, which is homeomorphic to $\mathbb{T}^{2}$.

The case $j(\Sigma) \geq 3$ requires more work. We already know how to proceed to find a dense region $O^{\prime} \subset S$ embeddable in $N_{3}$ and having two crossing circles. We next show how to take off a nonorientable circle from $O^{\prime}$ so than the resultant region $O$ (which is still dense in $S$ ) still has two crossing circles but can now be embedded in $\mathbb{T}^{2}$.

First we need to find an appropriate description for $N_{3}$. Let $M_{2} \subset \mathbb{R}^{3}$ be chosen in such a way that $x \in M_{2}$ implies $-x \in M_{2}$ as well. Let " $\approx$ " denote the equivalence relation in $M_{2}$ identifying $x$ and $-x$ for all $x \in M_{2}$. Then $N_{3} \cong M_{2} / \approx$ and we can assume in fact $O^{\prime} \subset N_{3}=M_{2} / \approx$. [Indeed it is clear that $M_{2} / \approx$ is a closed surface. To prove that it is nonorientable let $\pi$ : $M_{2} \rightarrow M_{2} / \approx$ denote the quotient map, fix points $x,-x \in M_{2}$ and construct an arc $\alpha \subset W$ having $x$ and $-x$ as its endpoints. It is not restrictive to assume that $\alpha$ contains no other pair of symmetrical points (otherwise take an open $\operatorname{arc} \beta \subset \alpha$ small enough having no such pairs, include it in a maximal open arc $\gamma \subset \alpha$ with the same property and replace $\alpha$ by the closure of $\gamma$ ). Clearly, there is a homeomorphism $h:[-1,1] \times(-1,1) \rightarrow U \subset M_{2}$ such that $h([-1,1] \times\{0\})=\alpha$ and $h(-1, t)=-h(1,-t)$ for any $t \in[-1,1]$. Then $\pi(\alpha)$ is a circle in $M_{2} / \approx$ having $\pi(U)$ (a Möbius band) as a neighbourhood and $\pi(\alpha)$ is nonorientable. Finally, $\chi\left(M_{2} / \approx\right)=\frac{\chi\left(M_{2}\right)}{2}=-1=\chi\left(N_{3}\right)$ is easy to prove by constructing a triangulation of $M_{2}$ such that if $K$ is any of its faces, edges or vertexes then $-K=\{-x: x \in K\}$ is also a face, an edge or a vertex of the triangulation.] 
It turns out that $\pi^{-1}\left(O^{\prime}\right)$ is connected. This follows from the fact that if $V \subset N_{g}$ is an arbitrary region and $U$ is one of the components of $\pi^{-1}(V)$ then $\left.\pi\right|_{U}$ is surjective, and hence we have that either $\pi^{-1}(V)$ is connected or $\pi^{-1}(V)$ consists of exactly two components, homeomorphic to $V$, and symmetrical each other: in the particular case $O^{\prime}=V$, the nonorientability implies that the first possibility holds. [To prove the surjectivity of $\left.\pi\right|_{U}$ argue by contradiction to find a boundary point $b$ of $\pi(U)$ in $V$ (because $V$ is connected). Notice that, since $\pi(U)$ is open (because $\pi$ is an open map), $b \notin \pi(U)$. Find a sequence $\left(y_{n}\right)_{n}$ of points of $\pi(V)$ converging to $b$ and take points $x_{n}$ in $U$ with $\pi\left(x_{n}\right)=y_{n}$; it is not restrictive to assume that $\left(x_{n}\right)_{n}$ converges, say to $a$. Since $\pi(a)=b, a \notin U$. Now it suffices to take a very small connected neighbourhood $U_{a}$ of $a$ and note that $\pi\left(U \cup U_{a}\right) \subset V$, contradicting the definition of $U$.]

Now it is easy to prove that if $\sigma, \tau \subset O^{\prime}$ are crossing circles then $\pi^{-1}(\sigma)=$ $\sigma_{1} \cup \sigma_{2}$ and $\pi^{-1}(\tau)=\tau_{1} \cup \tau_{2}$, where $\sigma_{l}$ and $\tau_{l}$ are crossing circles, $l=1,2$, and $\left(\sigma_{1} \cup \tau_{1}\right) \cap\left(\sigma_{2} \cup \tau_{2}\right)=\emptyset$. Clearly, $\pi^{-1}\left(O^{\prime}\right) \backslash\left(\sigma_{1} \cup \tau_{1} \cup \sigma_{2} \cup \tau_{2}\right)$ is then connected, so we can construct in it a (minimal) arc $\delta$ connecting two symmetrical points $x,-x \in O^{\prime}$. Now $\alpha=\pi(\delta)$ is a nonorientable circle and $\sigma, \tau \subset O:=O^{\prime} \backslash \alpha$. According to Lemma 3.2(i), $O$ is embeddable either in $\mathbb{B}^{2}$ or in $\mathbb{T}^{2}$. The existence in $O$ of crossing circles excludes the first possibility.

STEP 2: Constructing the homeomorphism to $\mathbb{T}^{2} \backslash A$.

Apply Theorem 3.1 to the surface $O$ to find the corresponding embedding $e^{\prime \prime}: O \rightarrow \mathbb{S}_{\Sigma^{\prime \prime}}^{2}$. Since $O$ is orientable we have $j\left(\Sigma^{\prime \prime}\right)=0$, since $O$ has two crossing circles we have $i\left(\Sigma^{\prime \prime}\right) \geq 1$, and since $O$ is embeddable in the torus we have $i\left(\Sigma^{\prime \prime}\right) \leq 1$ (otherwise $O$ would have two pairs of pairwise disjoint crossing circles, and since each of these circles is orientable and non-null homotopic - cf. Remark 3.3- we would apply Lemma 3.2(ii) to arrive a contradiction). Thus $\mathbb{S}_{\Sigma^{\prime \prime}}^{2} \cong \mathbb{T}^{2}$ and we can assume $O \subset \mathbb{T}^{2}$ and that $C=\mathbb{T}^{2} \backslash O$ is totally disconnected.

Denote by $\alpha$ and $\beta$ the crossing circles in $O$. Fix an annular neighbourhood $B \subset O$ of $\beta$ intersecting $\alpha$ at exactly a small open arc $\gamma$. Use Lemma 3.2(ii) to find a homeomorphism $f: \mathbb{T}^{2} \backslash \alpha \rightarrow \mathbb{S}^{1} \times(-1,1)$ such that, if $U$ is a closed annulus which is decomposed by $\alpha$ into two closed annuli $U_{l}$, $l=-1,1$, then the restriction of $f$ to $U_{l} \backslash \alpha$ can be extended to a homeomorphism $f_{l}: U_{l} \rightarrow f\left(U_{l} \backslash \alpha\right) \cup\left(\mathbb{S}^{1} \times\{l\}\right), l=-1,1$. Notice that the circles $\mathbb{S}^{1} \times\{l\}=f_{l} \circ \alpha=: \alpha_{l}$ have compatible orientations in $\mathbb{S}^{1} \times[-1,1]$ (because the disk $f(B \backslash \gamma)$ connects them).

Take into account that, because of [8, p. 539, Th. 5], any totally disconnected compact subset of $\mathbb{S}^{2}$ is included into some arc, that is, a homeomorphic set to a compact interval. Further, for any two given arcs in $\mathbb{S}^{2}$ there is a homeomorphism of the sphere mapping one of them onto the 
other one $[8$, p. 535 , Th. 1]. Combining these facts it is easy to construct a circle $\mu$ including $f(C)$ and separating $\mathbb{S}^{1} \times[-1,1]$ into two closed annuli: let us denote them by $P_{-1}$ and $P_{1}$. We can assume that $\alpha_{l}$ and $\mu$ have compatible orientations in $P_{l}, l=-1,1$. Then we can construct a homeomorphism $p$ from $\mathbb{S}^{1} \times[-1,1]$ into itself such that $p(t, l)=\alpha_{l}(t), l=-1,1$, and $p(t, 0)=\mu(t)$ for any $t \in \mathbb{S}^{1}$.

Fix now in $\mathbb{S}^{1} \times(-1,1)$ an arc $\delta$ of constant (say $\rho$ ) irrational slope, include it in a circle $\phi$ separating $\mathbb{S}^{1} \times[-1,1]$ into closed annuli $Q_{l}, l=-1,1$, and, as before, find a homeomorphism $q$ from $\mathbb{S}^{1} \times[-1,1]$ into itself leaving its boundary invariant and mapping $\mathbb{S}^{1} \times\{0\}$ onto $\phi$. Clearly, it is not restrictive to assume that $r=q \circ p^{-1}$ maps $f(C)$ into $\delta$. Notice also that $\left(r \circ f_{l} \circ \alpha\right)(t)=\left(r \circ \alpha_{l}\right)(t)=(t, l)$ for any $t \in \mathbb{S}^{1}$ and $l=-1,1$. Let $A=(r \circ f)(C)$. Then it is clear that $O$ is homeomorphic to $\mathbb{T}^{2} \backslash A$ (now we are seeing $\mathbb{S}^{1} \times(-1,1)$ as a subset of $\left.\mathbb{T}^{2}\right)$.

STEP 3: Finishing the proof.

Consider the irrational flow of slope $\rho$ in $\mathbb{T}^{2}$, take a diffeomorphic copy $M_{1} \subset \mathbb{R}^{3}$ of $\mathbb{T}^{2}$ and carry this flow to a smooth flow in $M_{1}$ with associated vector field $F: M_{1} \rightarrow \mathbb{R}^{3}$. Multiply $F$ by a smooth nonnegative function $\lambda: M_{1} \rightarrow[0, \infty)$ such that the resulting vector field has an associated flow which, after being carried to $\mathbb{T}^{2}$, has $A$ as its set of singular points. Use Theorem 2.1 to construct a smooth transitive flow on $O$ and extend it to a smooth flow $\Phi$ on the whole $S$ by means of Lemma 2.3. This concludes the proof of (iii) $\Rightarrow$ (iv) and Theorem A.

\section{Proof of Theorem B}

The idea of the proof is fairly simple. The "bricks" of our construction are compact cylinders $C \subset M$ ("blocks") with pairwise disjoint interiors, which will be useful to parametrize in order to fix their tops $U(C)$, their bottoms $R(C)$, and their open fibres ("vertical" curves in Int $C$ connecting one point from $U(C)$ with another one from $R(C)$ ). Starting from a "basement" $B$, we successively add "flats" (blocks whose bottom is included in the top of a previous flat or the basement) and "stairs" (blocks whose bottom is also included in the top of some flat or the basement and now with its top included in the bottom of the basement) to get an "infinite tower" dense in $M$. This will be done in such a way that the open fibres of the blocks are (not necessarily maximal) orbits of an appropriate flow and, moreover, one of the orbits of this flow (a countable union of open fibres from all the blocks and points from the corresponding tops and bottoms) is dense in $M$.

Throughout the proof we will assume, without loss of generality (cf. Lemma 2.3), that $M$ has empty boundary. 
Recall that a set $C \subset M$ is called an $n$-cell if it is homeomorphic to the closed ball $\mathbb{D}^{n}$. If additionally $\theta:[-1,1] \times \mathbb{D}^{n-1} \rightarrow C$ is a homeomorphism then we will call the pair $(C, \theta)$ a parametrized $n$-cell. We will write $R(C)=$ $R(C, \theta)=\theta\left(\{-1\} \times \mathbb{D}^{n-1}\right)$ and $U(C)=U(C, \theta)=\theta\left(\{1\} \times \mathbb{D}^{n-1}\right)$, and the arcs $\theta([-1,1] \times\{z\})\left(z \in \mathbb{D}^{n-1}\right)$ will be called the closed fibres of $C$ (with respect to the parametrization $\theta)$. If in these last definitions we replace $\mathbb{D}^{n-1}$ by $\mathbb{O}^{n-1}$ and $[-1,1]$ by $(-1,1)$ then we get $R_{O}(C)=R_{O}(C, \theta), U_{O}(C)=U_{O}(C, \theta)$ and the open fibres of $C$ (with respect to the parametrization $\theta$ ).

Let $\{(B, \beta)\} \cup\left\{\left(F_{i}, \phi_{i}\right)\right\}_{i=1}^{r} \cup\left\{\left(S_{j}, \sigma_{j}\right)\right\}_{j=1}^{s}$ be a finite collection of parametrized $n$-cells in $M$. We call this collection (which, if no confusion arises, we will identify with the union set $\left.T=B \cup \bigcup_{i=1}^{r} F_{i} \cup \bigcup_{j=1}^{s} S_{j}\right)$ a tower with basement $B=(B, \beta)$, flats $F_{i}=\left(F_{i}, \phi_{i}\right)$ and stairs $S_{j}=\left(S_{j}, \sigma_{j}\right)$ (we will generically refer to $B, F_{i}$ and $S_{j}$ as the blocks of $T$ ), provided that, for any block $C$, there is a nonnegative integer $l(C)$ (the level of $C$ ) such that the following properties hold:

(i) $l(B)=0$ and $l(C)>0$ for any other block $C$;

(ii) if $F$ is a flat of level $l$ then there is a flat $F^{*}$ of level $l-1$ such that $F \cap F^{*}=R(F) \subset U_{O}\left(F^{*}\right)$ (if $l=1$ then $F^{*}$ means the basement $B$ ); moreover, $F \cap C=\emptyset$ for any other block $C$ with $l(C) \leq l$;

(iii) if $S$ is a stair of level $l$ then $S \cap B=U(S) \subset R_{O}(B)$ and there is a flat $F^{*}$ of level $l-1$ such that $S \cap F^{*}=R(S) \subset U_{O}\left(F^{*}\right)$ (if $l=1$ then we mean $F^{*}=B$ and $S \cap F^{*}=R(S) \cup U(S)$ with $U(S) \subset R_{O}(B)$ and $\left.R(S) \subset U_{O}(B)\right)$; moreover, $S \cap C=\emptyset$ for any other block $C$.

Assume additionally that there is a number $0<\epsilon<1$ such that for any block $(C, \theta)$ of $T$ there is a continuous (or, if $M$ is smooth, a smooth) embedding extending $\theta, e(\theta):(-1-\epsilon, 1+\epsilon) \times \mathbb{O}^{n-1}(1+\epsilon) \rightarrow M$, and put $e(C):=e(\theta)\left((-1-\epsilon, 1+\epsilon) \times \mathbb{O}^{n-1}(1+\epsilon)\right)$. Moreover, assume that:

(iv) if $C$ and $C^{\prime}$ are disjoint blocks then $e(C)$ and $e\left(C^{\prime}\right)$ are disjoint as well;

(v) if $F=(F, \phi)$ and $F^{*}=\left(F^{*}, \phi^{*}\right)$ are as in (ii) then $e(\phi)\left(\{-1\} \times \mathbb{O}^{n-1}(1+\right.$ $\epsilon)) \subset U_{O}\left(F^{*}\right)$ and $e(\phi)(t, z)=e\left(\phi^{*}\right)\left(t+2, z^{*}\right)$ for any $t \in(-1-\epsilon,-1+\epsilon)$ whenever $z$ and $z^{*}$ are such that $e(\phi)(-1, z)=e\left(\phi^{*}\right)\left(1, z^{*}\right)$;

(vi) if $S=(S, \sigma)$ and $F^{*}=\left(F^{*}, \phi^{*}\right)$ are as in (iii) then $e(\sigma)(\{-1\} \times$ $\left.\mathbb{O}^{n-1}(1+\epsilon)\right) \subset U_{O}\left(F^{*}\right)$ and $e(\sigma)(t, z)=e\left(\phi^{*}\right)\left(t+2, z^{*}\right)$ for any $t \in(-1-\epsilon,-1+\epsilon)$ whenever $z$ and $z^{*}$ are such that $e(\sigma)(-1, z)=$ $e\left(\phi^{*}\right)\left(1, z^{*}\right)$; also, $e(\sigma)\left(\{1\} \times \mathbb{O}^{n-1}(1+\epsilon)\right) \subset R_{O}(B)$ and $e(\sigma)(t, w)=$ $e(\beta)\left(t-2, w^{*}\right)$ for any $t \in(1-\epsilon, 1+\epsilon)$ whenever $w$ and $w^{*}$ are such that $e(\sigma)(1, w)=e(\beta)\left(-1, w^{*}\right)$. 
Then we will call $T$ a regularizable tower and also write $e(T)=e(B) \cup$ $\bigcup_{i=1}^{r} e\left(F_{i}\right) \cup \bigcup_{j=1}^{s} e\left(S_{j}\right)$. The fibres of $T$ are the maximal path-connected subsets of Int $T$ consisting of a countable union of open or closed fibres of any of the blocks which the towers $T_{m}$ are made of. See Figure 2.

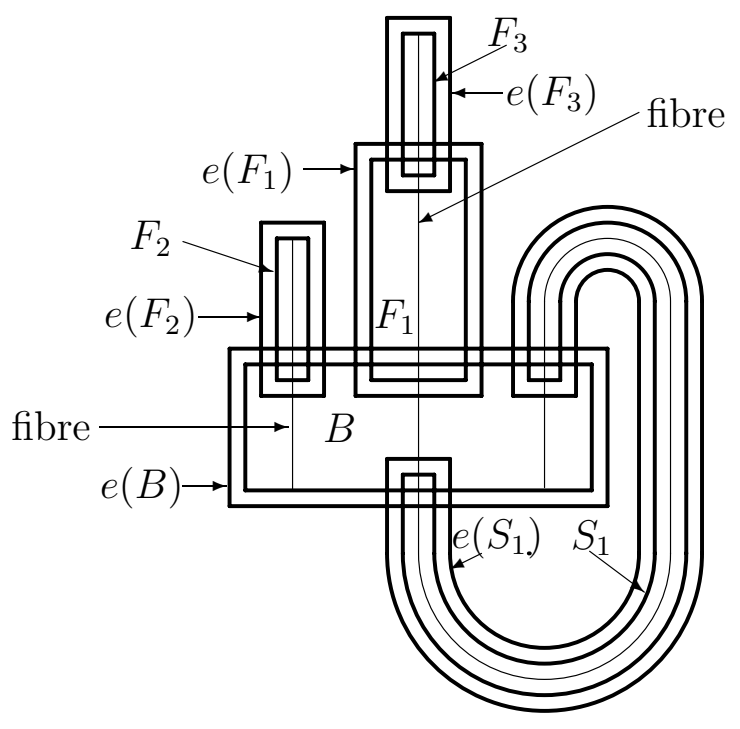

Figure 2: Regularizable tower with basement $B$, flats $F_{1}, F_{2}$ and $F_{3}$ and stair $S_{1}$ for which $l(B)=0, l\left(F_{1}\right)=l\left(F_{2}\right)=l\left(S_{1}\right)=1$ and $l\left(F_{3}\right)=2$.

Finally, we call $T$ an infinite regularizable tower if $T=\bigcup_{m=0}^{\infty} T_{m}$, where all $T_{m}$ are regularizable towers such that $T_{0}=\{(B, \beta)\}$ and, for any $m \geq 1$, there is a block $\left(C_{m}, \theta_{m}\right)$ of $T_{m}$ such that $T_{m}=T_{m-1} \cup\left\{\left(C_{m}, \theta_{m}\right)\right\}$. The fibres of $T$ are similarly defined as before.

We intend to construct an infinite regularizable tower $T$ dense in $M$ containing a dense fibre. The hardest part of the process consists of describing how to add to a given regularizable tower a new block containing (in an appropriate place) a prescribed point outside the initial tower. Next proposition pursues this aim:

Proposition 4.1. Let $T \subset M$ be a regularizable tower (we keep the notation above). Let $x \in U_{O}(F)$ for some flat $F$ of $T$ of level $l \geq 0$ (if $l=0$ then we mean $F=B$ ) and assume that $x \notin C$ for any other block $C$ of $T$.

(a) Let $y \in M \backslash T$. Then there is a regularizable tower $T^{*}=T \cup\left\{\left(F^{*}, \phi^{*}\right)\right\}$ for which $F^{*}$ is a flat of level $l+1$ and such that $x=\phi^{*}(-1, \mathbf{0})$, $y=\phi^{*}(1,0)$.

(b) Let $y \in R_{O}(B)$. Then there is a regularizable tower $T^{*}=T \cup\left\{\left(S^{*}, \sigma^{*}\right)\right\}$ for which $S^{*}$ is a stair of level $l+1$ and such that $x=\sigma^{*}(-1, \mathbf{0})$, $y=\sigma^{*}(1, \mathbf{0})$. 
We will divide the proof into two steps. First we will assume that $M$ is just a topological manifold; then we will deal with the smooth case.

Proof of Proposition 4.1 (the continuous case). It is easy to show that $e(T) \backslash T$ is connected (here we need of course $n \geq 3$ ), and since $e(T)$ is an open neighbourhood of $T$ in $M$ we get that $M \backslash T$ is connected as well. Then we can use that $T$ is regularizable to easily construct an $\operatorname{arc} A$ having $x$ and $y$ as its endpoints so that $A \backslash\{x, y\} \subset M \backslash T$. Due to the compactness of $A$, there is a number $\delta>0$ small enough such that if $A^{\prime} \subset A$ is an arc whose diameter is less than $\delta$ then there is an open set $W$ in $M$, homeomorphic to $\mathbb{R}^{n}$, such that $A^{\prime} \subset W$.

We will begin by assuming that $\operatorname{diam} A<\delta$, fixing an open set $W$ homeomorphic to $\mathbb{R}^{n}$ including $A$, and proving Part (a) of the proposition.

Construct small disjoint $n$-cells $C_{x}$ and $C_{y}$ in $W$ containing respectively $x$ and $y$ in their boundaries $B_{x}$ and $B_{y}$. Indeed, we will take $C_{x}=e(\phi)([1,1+$ $\mu] \times D$ ) for a small number $\mu<\epsilon$ and a small closed ball $D \subset \mathbb{O}^{n-1}$ and assume that $e(\phi)(1, a)=x$ for the centre $a$ of $D$. Notice that we can also assume that both $B_{x}$ and $B_{y}$ are regularizable $n$-spheres, that is, there are open neighbourhoods $O_{x}$ and $O_{y}$ of $B_{x}$ and $B_{y}$ and homeomorphisms $h_{x}, h_{y}$ from $(-1,1) \times \mathbb{S}^{n}$ onto $O_{x}$ and $O_{y}$ such that $h_{x}\left(\{0\} \times \mathbb{S}^{n}\right)=B_{x}$, $h_{y}\left(\{0\} \times \mathbb{S}^{n}\right)=B_{y}$. If $e(\phi)(1+\mu, a)=x^{*}$, we can finally suppose that there is an arc $A^{*} \subset W$ connecting $x^{*}$ and $y$ and containing no other point from $C_{x} \cup C_{y} \cup T$.

Let $W_{\infty}$ denote the one-point compactification of $W$. As both $B_{x}$ and $B_{y}$ are regularizable, the annulus theorem $[10,7,13]$ guarantees the existence of a homeomorphism $h:[-1,1] \times \mathbb{S}^{n} \rightarrow W_{\infty} \backslash \operatorname{Int}\left(C_{x} \cup C_{y}\right)$ mapping $\{-1\} \times \mathbb{S}^{n}$ onto $B_{x}$, and $\{1\} \times \mathbb{S}^{n}$ onto $B_{y}$. It is then rutinary to construct closed $(n-1)$ cells $K_{x^{*}}$ and $K_{y}$ in $B_{x}$ and $B_{y}$ (with indeed $K_{x^{*}}=e(\phi)(\{1+\mu\} \times E$ ) for some smaller closed ball $E \subset D$ also centred in $a$ ), and a parametrized $n$-cell $\left(F^{+}, \phi^{+}\right)$in $W \backslash \operatorname{Int}\left(C_{x} \cup C_{y}\right)$, very close to $A^{*}$ (hence not intersecting $T$ ), satisfying $F^{+} \cap B_{x}=R\left(F^{+}\right)=K_{x^{*}}, F^{+} \cap B_{y}=U\left(F^{+}\right)=K_{y}$, and such that $\phi^{+}(-1, \mathbf{0})=x^{*}, \phi^{+}(1,0)=y$.

The next step is to parametrize $F^{++}=F^{+} \cup e(\phi)([1,1+\mu] \times E)$ with a map $\phi^{++}$such that $R\left(F^{++}\right)=K_{x}:=e(\phi)(\{1\} \times E)\left(\right.$ and still $\left.U\left(F^{++}\right)=K_{y}\right)$ and with the additional property that if $u \in K_{x^{*}}$ and $e(\phi)(1+\mu, z)=u$ for some $z \in E$ then there is a $z^{++} \in \mathbb{D}^{n-1}$ such that $\phi^{++}\left(t, z^{++}\right)=e(\phi)(2+t, z)$ for any $t \in[-1,-1+\mu]$. Certainly we can also assume $\phi^{++}(-1, \mathbf{0})=x$, $\phi^{++}(1, \mathbf{0})=y$.

Finally we just write $F^{*}=\phi^{++}\left([-1,1] \times \mathbb{D}^{n-1}(\nu)\right)$ for some $\nu<1$ and define $\phi^{*}(t, z)=\phi^{++}(t, z / \nu)$. Obviously $T^{*}=T \cup\left\{\left(F^{*}, \phi^{*}\right)\right\}$ is regularizable (notice that we can use the same extensions for the blocks from $T$ just restricting them for an appropriate $\epsilon^{+}<\epsilon$ ). 
This ends the proof of Part (a) of the proposition under the additional assumption that $\operatorname{diam} A<\delta$. The proof of Part (b) has no substantial novelties; one just has to proceed similarly with both $x$ and $y$, using the basement $B$ for $y$ as we previously did with $F$ and $x$.

In the general case, choose points $x=x_{0}, x_{1}, \ldots, x_{k}=y$ decomposing $A$ into arcs $A_{1}, \ldots, A_{k}$ with diameters less than $\delta$. Reason as in the proof of Part (a) above to construct a parametrized $n$-cell $\left(F_{1}^{*}, \phi_{1}^{*}\right)$ with $x_{0}=$ $\phi_{1}^{*}(-1, \mathbf{0}), x_{1}=\phi_{1}^{*}(1, \mathbf{0})$ and such that $T_{1}=T \cup\left\{\left(F_{1}^{*}, \phi_{1}^{*}\right)\right\}$ is a regularizable tower for which $F_{1}^{*}$ is a flat of level $l+1$. Notice that the construction allows us to additionally assume that $T_{1} \cap\left(A_{3} \cup \cdots \cup A_{k}\right)=\emptyset$. Further (replacing if necessary $A_{2}$ for a very close arc with the same endpoints and not intersecting the closed fibre in $F_{1}^{*}$ containing both $x_{0}$ and $x_{1}$, and then taking a thinner $\left.F_{1}^{*}\right)$, it is not restrictive to suppose that $\left(A_{2} \backslash\left\{x_{1}\right\}\right) \cap T_{1}=$ $\emptyset$. Thus we proceed similarly as before to extend $T_{1}$ via a flat $\left(F_{2}^{*}, \phi_{2}^{*}\right)$ of level $l+2$ with $x_{1}=\phi_{2}^{*}(-1, \mathbf{0}), x_{2}=\phi_{2}^{*}(1, \mathbf{0})$ to a regularizable tower $T_{2}=T_{1} \cup\left\{\left(F_{2}^{*}, \phi_{2}^{*}\right)\right\}$.

Reasoning in this way we can construct in $M$ a regularizable tower $T \cup$ $\left\{\left(F_{i}^{*}, \phi_{i}^{*}\right)\right\}_{i=1}^{k}$ (if we are proving Part (a)) or $T \cup\left\{\left(F_{i}^{*}, \phi_{i}^{*}\right)\right\}_{i=1}^{k-1} \cup\left\{\left(S^{*}, \sigma^{*}\right)\right\}$ (if we are proving Part (b)) such that $x_{i-1}=\phi_{i}^{*}(-1, \mathbf{0}), x_{i}=\phi_{i}^{*}(1, \mathbf{0})$ for any $i$ (when we mean $\phi_{k}^{*}=\sigma^{*}$ in Part (b)). Now, if we take a very small $\nu$ and define $E_{1}=\mathbb{D}^{n-1}(\nu)$ and, inductively, $E_{i}$ for any $1<i \leq k$ by the property $\phi_{i}^{*}\left(\{-1\} \times E_{i}\right)=\phi_{i-1}^{*}\left(\{1\} \times E_{i-1}\right)$, it is clear that $\bigcup_{i=1}^{k} \phi_{i}^{*}\left(I \times E_{i}\right)$ is an appropriate choice for respectively $F^{*}$ or $S^{*}$ (the corresponding $\phi^{*}$ or $\sigma^{*}$ can be easily defined).

Proof of Proposition 4.1 (the smooth case). The basic ideas are analogous to those previously used (and we will maintain the notation above); only, in the part of the proof when $\operatorname{diam} A<\delta$ we have to proceed more carefully in order to guarantee differentiability. We next show how to do it (for Part (a) as before).

Notice first that if $W \subset M$ is the homeomorphic set to $\mathbb{R}^{n}$ such that $A \subset W$ we can assume, without loss of generality, that it is diffeomorphic to $\mathbb{R}^{n}$ as well.

Recall that $\lambda:=e(\theta):(1-\epsilon, 1+\epsilon) \times \mathbb{O}^{n-1}(1+\epsilon) \rightarrow M$ is now a smooth embedding and take as before $C_{x}=\lambda([1,1+\mu] \times D)$ for a small number $\mu<\epsilon$ and a small closed ball $D \subset \mathbb{O}^{n-1}$ with $\lambda(1, a)=x$ for the centre $a$ of $D$. Just to manage a symmetrical notation and stress the similarity of the argument for Part (b), we can assume that there is a smooth embedding $\gamma:(1-\epsilon, 1+\epsilon) \times \mathbb{O}^{n-1}(1+\epsilon) \rightarrow M$ such that $C_{y}:=\gamma([-1-\mu,-1] \times K)$ for a small closed ball $K \subset \mathbb{O}^{n-1}$ whose centre $b$ satisfies $\gamma(-1, b)=y$. We will assume that the radius of both $D$ and $K$ is $\mu$, that $\lambda([1-\mu, 1+\mu] \times D)$ and 
$\gamma([-1-\mu,-1+\mu] \times K)$ are disjoint and included in $W$ and that $\gamma([-1-$ $\mu,-1+\mu] \times K)$ does not intersect $T$. In what follows we will identify $W$ to $\mathbb{R}^{n}$ (thus $T^{\prime}:=T \cap W$ will be seen as a subset of $\mathbb{R}^{n}$ ); as the ensuing argument will make clear, it is not restrictive to do it so.

Consider the curves $\alpha:[1-\mu, 1+\mu] \rightarrow \mathbb{R}^{n}$ and $\eta:[-1-\mu,-1+\mu] \rightarrow \mathbb{R}^{n}$ respectively defined by $\alpha(t)=\lambda(t, a)$ and $\eta(t)=\gamma(t, b)$. Notice that, since $\lambda$ and $\gamma$ are diffeomorphisms, we have $\alpha^{\prime}(t) \neq \mathbf{0}$ and $\eta^{\prime}(t) \neq \mathbf{0}$ for any $t$. Now it is simple to connect them in a smooth way, that is, there is an injective smooth map $\kappa:[-1-\mu, 1+\mu] \rightarrow \mathbb{R}^{n}$ satisfying $\kappa^{\prime}(t) \neq \mathbf{0}$ for any $t, \kappa(t)=\alpha(t+2)$ for any $t \in[-1-\mu,-1+\mu]$, and $\kappa(t)=\eta(t-2)$ for any $t \in[1-\mu, 1+\mu]$. We can additionally assume that $\kappa([-1+\mu, 1-\mu]) \subset \mathbb{R}^{n} \backslash T^{\prime}$ and that

$$
\begin{aligned}
\kappa([-1+\mu, 1-\mu]) \cap \lambda([1-\mu, 1+\mu / 2] \times D) & =\emptyset, \\
\kappa([-1+\mu, 1-\mu]) \cap \gamma([-1-\mu / 2,-1+\mu] \times K) & =\emptyset .
\end{aligned}
$$

From [5, p. 69, Proposition 1.2] $\kappa^{\prime}(t) /\left\|\kappa^{\prime}(t)\right\|$ cannot map the interval [-1$\mu, 1+\mu]$ onto $\mathbb{S}^{n}$, that is, there is a point $p \in \mathbb{S}^{n}$ such that $\kappa^{\prime}(t) /\left\|\kappa^{\prime}(t)\right\| \neq p$ for any $t$. Fix a diffeomorphism $g: \mathbb{R}^{n-1} \rightarrow \mathbb{S}^{n-1} \backslash\{p\}$, and define $G_{r}$ : $\mathbb{S}^{n-1} \backslash\{p\} \rightarrow \mathbb{R}^{n}, 1 \leq r \leq n-1$, by

$$
G_{r}(q)=\frac{\partial g}{\partial z_{r}}\left(g^{-1}(q)\right)
$$

where $z_{r}$ denotes the $r$-th component in $\mathbb{R}^{n-1}$. As all vectors $G_{r}\left(\kappa^{\prime}(t) /\left\|\kappa^{\prime}(t)\right\|\right)$ are ortogonal to $\kappa^{\prime}(t),\left\{\kappa^{\prime}(t), G_{1}\left(\kappa^{\prime}(t) /\left\|\kappa^{\prime}(t)\right\|\right), \ldots, G_{n-1}\left(\kappa^{\prime}(t) /\left\|\kappa^{\prime}(t)\right\|\right)\right\}$ is a base for any $t$ and we can assume, provided that $\mu^{*}<\mu$ is small enough, that $\xi:(-1-\mu, 1+\mu) \times \mathbb{O}^{n-1}\left(\mu^{*}\right) \rightarrow \mathbb{R}^{n}$ given by

$\xi\left(t, z_{1}, \ldots, z_{n-1}\right)=\kappa(t)+z_{1} G_{1}\left(\kappa^{\prime}(t) /\left\|\kappa^{\prime}(t)\right\|\right)+\cdots+z_{n-1} G_{n-1}\left(\kappa^{\prime}(t) /\left\|\kappa^{\prime}(t)\right\|\right)$

is an embedding and

$$
\begin{aligned}
\xi\left([-1+\mu / 4,1-\mu / 4] \times \mathbb{D}^{n-1}\left(\mu^{*} / 2\right)\right) \cap \lambda([1-\mu, 1+\mu / 8] \times D) & =\emptyset, \\
\xi\left([-1+\mu / 4,1-\mu / 4] \times \mathbb{D}^{n-1}\left(\mu^{*} / 2\right)\right) \cap \gamma([-1-\mu / 8,-1+\mu] \times K) & =\emptyset .
\end{aligned}
$$

Let $\rho_{c, d, s}: \mathbb{R}^{n} \rightarrow \mathbb{R}, d>c>0, s>0$, be a nonnegative smooth map satisfying $\rho_{c, d, s}(t, z)=1$ for any $(t, z) \in[-c, c] \times \mathbb{D}^{n-1}(s / 2)$ and vanishing exactly at $\mathbb{R}^{n} \backslash(-d, d) \times \mathbb{O}^{n-1}(s)$, and construct in $\mathbb{R}^{n}$ the vector fields defined by

$$
\begin{aligned}
& X(\lambda(t, z))=\rho_{\mu / 4, \mu / 2, \mu}(t-1, z-a) \frac{\partial \lambda}{\partial t}(t, z), \\
& Y(\gamma(t, z))=\rho_{\mu / 4, \mu / 2, \mu}(t+1, z-b) \frac{\partial \gamma}{\partial t}(t, z), \\
& Z(\xi(t, z))=\rho_{1-\mu / 2,1-\mu / 4, \mu^{*} / 2}(t, z) \frac{\partial \xi}{\partial t}(t, z),
\end{aligned}
$$


whenever the expressions above make sense and zero elsewhere. Clearly, they all are well defined and smooth. Moreover, let $H=X+Y+Z$. As $H$ vanishes outside a bounded subset of $\mathbb{R}^{n}$, its associated flow $\Phi$ is well defined as well. Notice that, because of $(4.1)$, the arc $\kappa([-1-\mu, 1+\mu])$ is included in one of the orbits of $\Phi$, and, because of (4.2), we have

$$
\begin{aligned}
& \Phi(t, \lambda(1, z))=\lambda(t+1, z), \quad|t| \leq \mu / 8, z \in D, \\
& \Phi(t, \gamma(-1, z))=\gamma(t-1, z), \quad|t| \leq \mu / 8, z \in K \text {. }
\end{aligned}
$$

Say $\Phi\left(t_{x}, x\right)=y\left(\right.$ thus $\left.\Phi_{x}\left(\left[0, t_{x}\right]\right)=\kappa([-1,1])\right)$ and define $\varphi: \mathbb{R} \times$ Int $D \rightarrow \mathbb{R}^{n}$ by $\varphi(t, z)=\Phi(t, \lambda(1, z))$. We claim that $d \varphi(t, z)$ is regular (its determinant does not vanish) for any $(t, z)$. Indeed

$$
\frac{\partial \varphi}{\partial z_{r}}(t, z)=d \Phi_{t}(\lambda(1, z)) \frac{\partial \lambda}{\partial z_{r}}(1, z),
$$

$1 \leq r \leq n-1$, and

$$
\begin{aligned}
\frac{\partial \varphi}{\partial t}(t, z) & =\lim _{h \rightarrow 0} \frac{\Phi_{t}(\Phi(h, \lambda(1, z)))-\Phi_{t}(\Phi(0, \lambda(1, z)))}{h} \\
& =\left.\frac{\partial}{\partial h} \Phi_{t}(\Phi(h, \lambda(1, z)))\right|_{h=0} \\
& =d \Phi_{t}(\lambda(1, z)) \frac{\partial \varphi}{\partial t}(0, z)=d \Phi_{t}(\lambda(1, z)) \frac{\partial \Phi}{\partial t}(0, \lambda(1, z)) \\
& =d \Phi_{t}(\lambda(1, z)) H(\lambda(1, z))=d \Phi_{t}(\lambda(1, z)) \frac{\partial \lambda}{\partial t}(1, z) .
\end{aligned}
$$

The claim follows from the fact that both $\lambda$ and $\Phi_{t}$ are diffeomorphisms.

Let $\mu^{* *}>0$ be so small a number that $\gamma^{-1} \circ \varphi$ is well defined on $\left(t_{x}-\right.$ $\left.\mu^{* *}, t_{x}+\mu^{* *}\right) \times O^{* *}$, with $O^{* *}$ the open disk of radius $\mu^{* *}$ centred in $a$, and let $\tau$ denote the first component of $\gamma^{-1}$. Then

$$
\begin{aligned}
\frac{\partial(\tau \circ \varphi)}{\partial t}\left(t_{x}, a\right) & =\nabla \tau\left(t_{x}, a\right) \cdot \frac{\partial \varphi}{\partial t}\left(t_{x}, a\right) \\
& =\nabla \tau\left(t_{x}, a\right) \cdot H\left(\Phi\left(t_{x}, \lambda(1, a)\right)\right) \\
& =\nabla \tau(y) \cdot H(y) \\
& =\nabla \tau(y) \cdot \frac{\partial \gamma}{\partial t}(-1, b) \\
& =1
\end{aligned}
$$

and (provided that $\mu^{* *}$ is small enough) we can apply the implicit function theorem to find a smooth map $t=t(z)$ satisfying $t(a)=t_{x}$ and $\varphi(t(z), z) \in$ $\gamma(-1, K)$ for any $z \in O^{* *}$. 
Let $f_{1}, f_{2}: \mathbb{R} \rightarrow \mathbb{R}$ be nondecreasing smooth maps satisfying, respectively:

$$
\begin{aligned}
& f_{1}(t)=t, t \in\left(-\infty, \frac{\mu}{8}\right] ; f_{1}(t)=\frac{\mu}{4}, t \in\left[\frac{\mu}{4}, \infty\right) ; f_{1}^{\prime}(t)>0, t \in\left(-\infty, \frac{\mu}{4}\right) ; \\
& f_{2}(t)=0, t \in(-\infty, 0] ; \quad f_{2}(t)=2-\frac{\mu}{2}, t \in[1, \infty) ; \quad f_{2}^{\prime}(t)>0, t \in(0,1),
\end{aligned}
$$

and define

$$
f(t, z)=f_{1}(t)+f_{2}\left(\frac{t-\mu / 8}{t(z)-\mu / 4}\right)+\mu / 4-f_{1}(t(z)-t) .
$$

As $\varphi(t, z)$ is a local diffeomorphism, it is rutinary to check (again if $\mu^{* *}$ is sufficiently small) that the map $\varphi^{*}:(-\mu / 8,2+\mu / 8) \times O^{* *} \rightarrow \mathbb{R}^{n}$ given by

$$
\varphi^{*}(f(t, z), z)=\varphi(t, z)
$$

is a well defined diffeomorphism, satisfying for any $z \in O^{* *}$

$$
\varphi^{*}(2, z) \in \gamma(\{-1\} \times K)
$$

and (due to (4.3))

$$
\begin{array}{rlrl}
\varphi^{*}(t, z) & =\lambda(t-1, z), & & |t| \leq \mu / 8 \\
\varphi^{*}(t+2, z)=\gamma(t-1, w) & & (\text { if } z \text { and } w \text { are such that } \varphi(2, z)=\gamma(-1, w)), \\
& |t| \leq \mu / 8
\end{array}
$$

Now we define $\phi^{*}(t, z)=\varphi^{*}\left(t+1,2(z-a) / \mu^{* *}\right)$ and $F^{*}=\phi^{*}\left([-1,1], \mathbb{D}^{n-1}\right)$ and we are done.

Proof of Theorem B. Using Proposition 4.1 we can construct an infinite regularizable tower $T$ in $M$ with the additional property that one of its fibres, $\varrho^{*}$, is dense in $M$. More precisely, fix a dense sequence of points $\left\{u_{m}\right\}_{m=0}^{\infty}$ in $M$. Then it suffices to construct inductively (see the next paragraph) the corresponding regularizable towers $T_{m}$ with common basement $B$ and union the desired $T$ so that all fibres of all $T_{m}$ are open arcs (thus their closures are arcs), and there are fibres $\varrho_{m}$ of $T_{m}$ such that, for any $m$,

(*) neither of the endpoints of $\mathrm{Cl} \varrho_{m}$ belong to the intersection of two blocks of $T_{m}$,

(**) $\varrho_{m} \subset \varrho_{m+1}$,

$(* * *) d\left(\varrho_{m}, u_{m}\right)<1 / 2^{m}$

because then $\varrho^{*}:=\bigcup_{m=0}^{\infty} \varrho_{m}$ would do the job. 
It is worth emphasizing that if $\alpha$ is an arbitrary fibre of some $T_{m}$ then one of the endpoints belongs to $R_{O}(B)$ and the other one belongs to $U_{O}(F)$ for some block (a flat or the basement) $F$ of $T_{m}$, and neither of them belong to another $R_{O}(C)$ or $U_{O}(C)$, with $C$ any block of $T_{m}$. Thus (*) means that if $z_{m} \in R_{O}(B)$ and $x_{m} \in U_{O}\left(F_{m}\right)$ are the endpoints of $\mathrm{Cl} \varrho_{m}$ then they do not belong to another $R(C)$ or $U(C)$.

The choosing of $T_{0}$ is trivial, as it is that of $T_{m+1}$ in the case when $u_{m+1} \notin T_{m}$ : just use Proposition 4.1 (a) to add a flat $(F, \phi)$ to $T_{m}$ so that $\phi(-1, \mathbf{0})=x_{m}$ and $\phi(1, \mathbf{0})=u_{m+1}$, when $\varrho_{m+1}:=\varrho_{m} \cup \phi([-1,1) \times\{\mathbf{0}\})$. If $u_{m+1} \in T_{m}$ then we find a fibre $\alpha$ of $T_{m}$ very close to $u_{m+1}$ with endpoints $z \in R_{O}(B)$ and $x \in U_{O}(F)$ so that neither of them belong to any other $R(C)$ or $U(C)$. The way to do this is first choosing a fibre $\alpha_{1}$ of $T_{m}$ just close enough to $u_{m+1}$, then (if necessary) a fibre $\alpha_{2}$ with its endpoint in $R_{O}(B)$ very close to that of $\alpha_{1}$ so that it belongs to $U(S)$ for no stair $S$ of $T_{m}$, and finally (if necessary) the fibre $\alpha$ with both of its endpoints very close to those of $\alpha_{2}$ and satisfying the required property. Then we apply Proposition $4.1(\mathrm{~b})$ to add a stair $(S, \sigma)$ to $T_{m}$ satisfying $\sigma(-1, \mathbf{0})=x_{m}$ and $\sigma(1, \mathbf{0})=z$, being $\varrho_{m+1}:=\varrho_{m} \cup \alpha \cup \sigma([-1,1] \times\{\mathbf{0}\})$. Observe that we must be careful in choosing $S$ thin enough so that all fibres of $T_{m}$ starting from $U(S)$ end in $U_{O}(F)$, and all fibres of $T_{m}$ ending at $R(S)$ start from points of $R_{O}(B)$ outside $U(S)$. In this way, we guarantee that all fibres from $T_{m+1}$ are open arcs.

Notice that if $\varrho$ is a fibre of $T$ and $u \in \varrho$ then there are an open interval $I_{u} \ni 0$ and a bijection $\varrho_{u}: I_{u} \rightarrow \varrho$ (both unambiguously defined) such that $\varrho_{u}(0)=u$ and having the following property: for any $v \in \varrho \cap e(C)$ for some block $(C, \phi)$ in $T, v=\varrho_{u}\left(t^{*}\right)=e(\phi)\left(t^{* *}, z\right)$, there is a small number $\epsilon_{v}>0$ such that $\varrho_{u}\left(t+t^{*}\right)=e(\phi)\left(t+t^{* *}, z\right)$ for all $|t|<\epsilon_{v}$. It is not difficult to realize that $\Phi(t, u):=\varrho_{u}(t)$ is a well defined local flow on $M$ (which is differentiable in the smooth case as, once we see $M$ as embedded in $\mathbb{R}^{2 n}$ and put with the notation above $F(v):=\frac{\partial e(\phi)}{\partial t}\left(t^{* *}, z\right)$, it is clear that the map $F: O \rightarrow \mathbb{R}^{2 n}$ so constructed is a well defined smooth vector field on $M$ whose associated local flow is precisely $\Phi)$. Apply Lemma 2.3 to finish the proof.

Remark 4.2. It must be stressed that, in a sense, the proof of Theorem B can be shortened as one could dispose of Part (b) of Proposition 4.1. Indeed, using just Part (a) of the proposition it is not hard to prove that $\mathbb{R}^{n}$ can be densely embedded in $M$, and now we only need to use a transitive flow in $\mathbb{R}^{n}$ as those of [15], [1] or [16].

Yet we think that our approach is preferable. On the one hand, it provides a self-contained proof of the transitivity of $\mathbb{R}^{n}$ which is rather simpler than those of [1] or [16]. Moreover, Part (b) generalizes (and thus brings 
into attention to the western readership) the beautiful ideas in Sidorov's paper [15]. Finally, when constructing a transitive flow one should ideally tried to devise it with a number of singular points as small as possible, as then one could have a better chance to derive a minimal flow from it: Part (b), up to some extent, "reduces" this number. In this context it is worth recalling that the very important problem of proving or disproving that $\mathbb{S}^{3}$ admits a minimal flow still remains open.

\section{References}

[1] Anosov, D. V.: Existence of smooth ergodic flows on smooth manifolds. Math. USSR-Izv. 8 (1974), 525-552.

[2] Bendixson, I.: Sur les courbes définies par des équations différentielles. Acta Math. 24 (1901), 1-88.

[3] BeniéRe, J.-C.: Feuilletage minimaux sur les surfaces non compactes. Ph.D. Thesis, Université Claude Bernard Lyon 1, 1998.

[4] Blohin, A. A.: Smooth ergodic flows on surfaces. Trans. Moscow Math. Soc. 27 (1972), 117-134.

[5] Hirsch, M. W.: Differential topology. Springer-Verlag, New York, 1988.

[6] Jiménez López, V. And Soler López, G.: Accumulation points of nonrecurrent orbits of surface flows. To appear in Topology Appl.

[7] Kirby, R. C.: Stable homeomorphisms and the annulus conjecture. Ann. of Math. 89 (1969), 575-582.

[8] Kuratowski, K.: Topology, Vol. II. Academic Press, New York-London, 1968.

[9] Markley, N. G.: The Poincaré-Bendixson theorem for the Klein bottle. Trans. Amer. Math. Soc. 135 (1969), 159-165.

[10] Moise, E. E.: Affine structures in 3-manifolds. V. The triangulation theorem and Hauptvermutung. Ann. of Math. 56 (1952), 96-114.

[11] Munkres, J. R.: Topology, second edition. Prentice-Hall, Upper Saddle River, New Jersey, 2000.

[12] Oxtoby, J. C. And Ulam, S. M.: Measure-preserving homeomorphisms and metrical transitivity. Ann. of Math. 42 (1941), 874-920.

[13] Quinn, F.: Ends of maps. III. Dimensions 4 and 5. J. Differential Geom. 17 (1982), 503-521.

[14] Richards, I.: On the classification of noncompact surfaces. Trans. Amer. Math. Soc. 106 (1963), 259-269.

[15] Sidorov, Ye. A.: Smooth topologically transitive dynamical systems. Mat. Zametki 4 (1968), 751-759. (Russian)

[16] Smith, R. A. And Thomas, S.: Some examples of transitive smooth flows on differentiable manifolds. J. London Math. Soc. (2) 37 (1988), 552-568. 
[17] Smith, R. A. And Thomas, S.: Transitive flows on two-dimensional manifolds. J. London Math. Soc. (2) 37 (1988), 569-576.

[18] Soler López, G.: $\omega$-límites de sistemas dinámicos continuos. Master Thesis, Universidad de Murcia, 1999.

[19] Thurston, W. P.: Three dimensional geometry and topology. Princeton Mathematical Series 35. Princeton University Press, Princeton, N.J., 1997.

Recibido: 19 de febrero de 2002

Revisado: 23 de julio de 2002

Víctor Jiménez López Departamento de Matemáticas

Universidad de Murcia Campus de Espinardo, 30100 Murcia, Spain

vjimenez@um.es

Gabriel Soler López

Departamento de Matemática Aplicada y Estadística Universidad Politécnica de Cartagena

Paseo Alfonso XIII, 52

30203 Cartagena, Spain

gabriel.soler@upct.es

This paper has been partially supported by the DGISEC, grant PB98-0374-C03-01, by Fundación Séneca (Comunidad Autónoma de la Región de Murcia, Spain), grant PI-8/00807/FS/01, and MCYT and FEDER, grant BFM2002-03512. 